\title{
Research Paper: Effects of Lower Limbs Kinesio Taping on Balance Ability in Children With Cerebral Palsy: A Pilot Randomized Clinical Trial
}

Mirjavad Tabatabaee $^{1}$ (D), Alireza Shamsoddini ${ }^{1 *}$ (i), Moslem Cheraghifard ${ }^{2}$ (i)

1. Exercise Physiology Research Center, Baqiyatallah University of Medical Sciences, Tehran, Iran.

2. Department of Occupational Therapy, School of Rehabilitation Sciences, Iran University of Medical Science, Tehran, Iran.

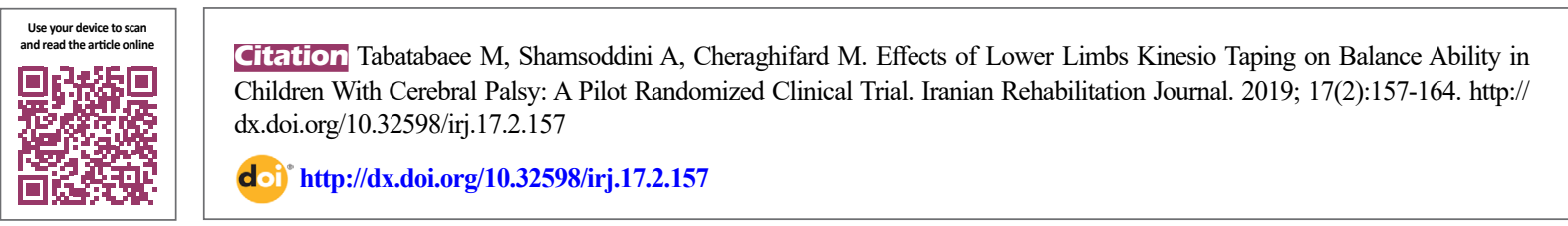

Article info:

Received: 12 Aug 2018

Accepted: 23 Jan 2019

Available Online: 01 June 2019

Keywords:

Cerebral Palsy, Postural

balance, Kinesio tape

\section{ABSTRACT}

Objectives: Cerebral Palsy (CP) is a non-progressive brain damage that leads to movement and balance disorders. One of the new rehabilitation approaches for such children is Kinesio taping. The present study aimed to investigate the effect of lower limbs kinesio taping on static and dynamic balance in children with $\mathrm{CP}$.

Methods: In this pilot randomized clinical trial, 30 children with CP were randomly classified into two control and intervention groups each containing 15 subjects. In the intervention group, Kinesio tape was applied with $30 \%$ tension on anterior muscles of lower limbs. However, in the control group, Kinesio tape was used just in a sham mode. In both groups, Kinesio tape was used along with occupational therapy interventions for two weeks. Berg Balance Scale (BBS) and forward Functional Reach (FR) tests were used before, two days, and two weeks after intervention. Two-way repeated measures ANOVA was used to study the intervention effects, within-group data were analyzed with the paired t-test, and between-group effects were assessed using independent t-test.

Results: Short term results (two days after intervention) indicated no significant difference between the scores of BBS $(\mathrm{P}=0.7)$ and $\mathrm{FR}(\mathrm{P}=0.08)$ in the intervention group, and $\mathrm{BBS}(\mathrm{P}=$ $0.8)$ and $\mathrm{FR}(\mathrm{P}=0.1)$ in the control group. Long term results indicated a significant change in BBS $(\mathrm{P}<0.001)$ and FR $(\mathrm{P}<0.001)$ in the intervention group, however, no significant changes were observed in $\mathrm{FR}(\mathrm{P}=0.1)$ and $\mathrm{BBS}(\mathrm{P}=0.8)$ in the control groups.

Discussion: Application of Kinesio tape on lower limbs of children with CP improved their balance. Therefore, this method can be used in rehabilitation clinics for functional improvement of children with Cerebral Palsy.

\section{* Corresponding Author:}




\section{Highlights}

- Lower-limb Kinesio taping can improve balance ability in children with Cerebral Palsy.

- Long-term application of Kinesio taping is more effective for improving balance than its short-term use.

\section{Plain Language Summary}

Cerebral Palsy (CP) is a group of permanent movement disorders that appear in early childhood. CP is caused by abnormal development or damage to some parts of the brain that control movement, balance, and posture. One contributing factor to children's problems with gait and reaching movement is poor balance control ability. Kinesio taping technique is a relatively new technique applied in rehabilitation programs of CP. The present study aimed to investigate the effect of lower-limb Kinesio taping on the balance ability of children with CP. The result shows that lower-limb Kinesio taping can improve balance ability in children with CP, especially its long-term application.

\section{Introduction}

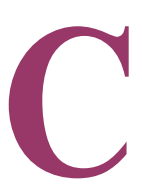

erebral palsy is defined as a non-progressive brain damage caused by abnormal brain development and brain injuries before, during, or after birth [1, 2]. Statistics indicate that $70 \%$ to $80 \%$ of patients with

Cerebral Palsy have spastic type of it; they experience movement and balance problems [3, 4]. In children with Cerebral Palsy, altered muscle function causes balance problems, making it difficult for the person to stand and walk [4]. Due to permanent tension of muscles in such patients, they have permanent spasticity.

These conditions along with other complications make a kind of immobility. Therefore, muscular function and strength do not have a linear relationship with aging and gaining weight [5]. Since balance is the last stage of cortical growth in children, spastic Cerebral Palsy is followed by more balance and movement disorders [3, $6,7]$. Balance has two static and dynamic modes. Static balance is referred to the ability of body in maintaining and controlling center of gravity within a specifically supported range and keeping its standing position via feet [8-10]. Weak static balance causes movement limitations followed by reduced ability of understanding the surroundings, communicating with family members and friends [11-15].

Dynamic balance disorder creates functional problems for daily activities [16]. Successive falls caused by paresis and disability of the person to control posture fluctuations along with static and dynamic balance control are some prevalent complications of dynamic balance disorder [17]. Balance is one of the main components of movement and increases functional skills [18]. Reac- tions of static and dynamic balance are weaker in children with Cerebral Palsy [19]. Children with spastic Cerebral Palsy have abnormal muscle tone and status control, both of which affect functional balance [20].

Functional balance is one of the components of status stability that makes it possible for the children to act independently in all the daily and social activities in home, school, and society. Functional balance should be evaluated in order to understand whether the child is independent in his/her daily activities or not [21]. Children with Cerebral Palsy have low physical activity; therefore, they experience some disorders in their daily activities such as independent walking, climbing stairs, and running. Stature control and balance are significant components of functional movement in sitting and standing activities, and walking [22].

According to the significance of balance function and its effects on individual functions, communication with the society and independence, it can be said that balance disorders are considered as one of the most important challenges for the children and rehabilitation of such disorders is the main goal of physiotherapists and occupational therapists [23].

Kinesio tape is a new technique applied by an elastic tape called Kinesio Flex Tape [13]. This tape stimulates peripheral sensory system of the skin. This sensory system is related to pains, movement control, and proprioception [24] and can improve proprioception and weak muscles, and reduce edema and muscle spasms [25].

A case study by Losa (2015) indicated that using Kinesio tape is a positive forward step toward rehabilitation of children with Cerebral Palsy and can affect their static 
and dynamic balance [26]. Yasukawa claimed that Kinesio tape stimulates sensory-movement system and, consequently, voluntary control and coordination of physiotherapy programs of children with Cerebral Palsy is improved [12]. Sliwinsky (2007) indicated that Kinesio tape controls muscle tone and directly improves movement activities of the patients [27]. The studies showed that KT technique as part of a multimodal therapy program can affect the rehabilitation of children with $\mathrm{CP}$ to improve motor function and dynamics [28].

There are some limitations in such insufficient studies conducted on the balance and reduced life quality of the patients. But to date, no study specifically examined the effect of the kinesio taping on the dynamic and static balance of children with CP. Therefore, the present study aimed at investigating the effect of lower limbs kinesio taping on static and dynamic balance in children with Cerebral Palsy.

\section{Methods}

The current Randomized Clinical Trial (IRCT2017082135822N1) was conducted on 30 children with spastic diplegia and quadriplegia and Cerebral Palsy, aged 3-10 referred to Tavanafarin and Nasim rehabilitation clinics in Tehran, Iran, from November 2017 to May 2018 and subjects were randomly divided into intervention and control groups. The current study was approved by the Human Research Ethics Committee of the Baqiyatallah University of Medical Sciences (ethical code: IR.BMSU.REC.1396.526).

All patients' parents signed the informed consent form and offered the opportunity to ask any questions regarding the study procedures. Inclusion criteria of the study were: 1. Diagnosis of spastic diplegia and quadriplegia Cerebral Palsy by neurologist; 2. Minimum walking ability; 3. Ability of understanding and executing the orders; 4 . Age from 4 to 10 years old; 5 . No other medical interventions; and 6 . No genu recurvate.

The children receiving simultaneous interventions or with sensitivity toward taping were excluded from the study. The participants were classified into two 15-patient intervention and control groups. Then, the specified tests and evaluations were performed by the researcher in the clinic. Kinesio tape was used as a treatment tool in the intervention group, and as a sham tool in the control group. Moreover, both groups used physiotherapy and occupational treatment along with Kinesio tape. The researcher was trained by workshops to use Kinesio tapes.
Taping was applied on ankles and tibialis muscles to improve dorsi flexion, on rectus femoris to improve knee extension, and on hamstring muscles to inhibit knee flexion. Therefore, the applied tension was from the beginning to the end of muscle. However, in inhibitory actions, it was applied from the end toward the beginning of the muscle. Moreover, for tape muscle modification, the muscle was tensioned up to $30 \%$ of the primary length, and for function modification up to $100 \%$ of the primary length of the muscle [12].

In kinesio taping, the child bends slightly from the trunk to make in the paravertebral muscles stretch [26]. Taping was done for 14 days, 24 hours a day, on both feet, and was changed every four days. The tests and evaluations were performed in the intervals of before taping, two days after taping, and 14 days after taping, which meant at the end of interventions. Functional balance was tested via Berg balance scale including 14 items evaluated in an answer range of 0-4 for each item. Reliability of this test was reported $95 \%$ by Azad et al. [29].

Forward functional reach tests individual's balance while standing near a wall and stretches the hands. Therefore, the extent of stretching of the hand, evaluated by yardstick, was recorded. Duncan and Stevensky indicated that functional access test had a concurrent validity in balance evaluation [30].

\section{Data analyses}

Shapiro-wilk test was employed to evaluate normal distribution of the data. It was indicated that the variables of the current study had a normal distribution. To compare the variables of each group, paired t-test was used before and after intervention, and for comparison of intervention data in two different groups, independent t-test was used at two separate times. Also, the effects of time (before, after 2 days, at the end) and groups (intervention or control) and their interactive effects were estimated by two-way repeated measures ANOVA. Data were analyzed with SPSS V. 20 software $(\mathrm{P}<0.05)$.

\section{Results}

Totally, the current study recruited 30 patients with Cerebral Palsy (18 males and 12 females). Average age of participants was seven years in the intervention group, and 6.93 years in the control group. There were 10 male and five female subjects in the interventions group, and eight male and seven female subjects in the control group. In both groups, $53.3 \%$ of the participants had diplegia, and $47.7 \%$ quadriplegia. Table 1 indicates 
Table 1. Mean \pm SD of Berg Balance scale and functional reach in the two groups

\begin{tabular}{ccccc}
\hline \multirow{2}{*}{ Variable } & Group & \multicolumn{3}{c}{ Time } \\
\cline { 3 - 5 } & & Before & After 2 Days & After 2 Weeks \\
\hline \multirow{2}{*}{ Berg Balance Scale } & Intervention & $42.13 \pm 5.71$ & $42.20 \pm 5.34$ & $44.13 \pm 5.26$ \\
& Control & $39.40 \pm 3.86$ & $39.40 \pm 4.01$ & $39.40 \pm 4.01$ \\
\hline \multirow{2}{*}{ Functional reach } & Intervention & $18.66 \pm 2.66$ & $18.86 \pm 2.77$ & $20.60 \pm 2.50$ \\
& Control & $19.06 \pm 1.86$ & $19.06 \pm 1.98$ & $19.33 \pm 1.75$ \\
\hline
\end{tabular}

Iranian Rehabilitation Journal

Table 2. Summary of analysis of variance in the two groups

\begin{tabular}{cccc}
\hline Variables & Effects & $\mathbf{f}$ & $\mathbf{P}$ \\
\hline & Time & 20.88 & $<0.001$ \\
Berg Balance scale & Group & 0.392 & 0.058 \\
& Timexgroup & 20.88 & $<0.001$ \\
Functional reach & Time & 54.63 & $<0.001$ \\
& Group & 0.072 & 0.790 \\
& Timexgroup & 30.57 & $<0.001$ \\
\hline
\end{tabular}

Iranian Rehabilitation Journal

means and standard deviations of FR and BBS tests of both groups in different times. In the primary evaluation, result of independent t-test showed, no significance difference between FR scores of each group $(\mathrm{P}=0.6)$. It was the case for BBS scores as well $(\mathrm{P}=0.1)$.

Table 2 presents ANOVA results for the estimated variables. The main effects of time (before, after 2 days, and after two weeks) and interaction between time and group (group $\times$ time) were significant for both variables. How-

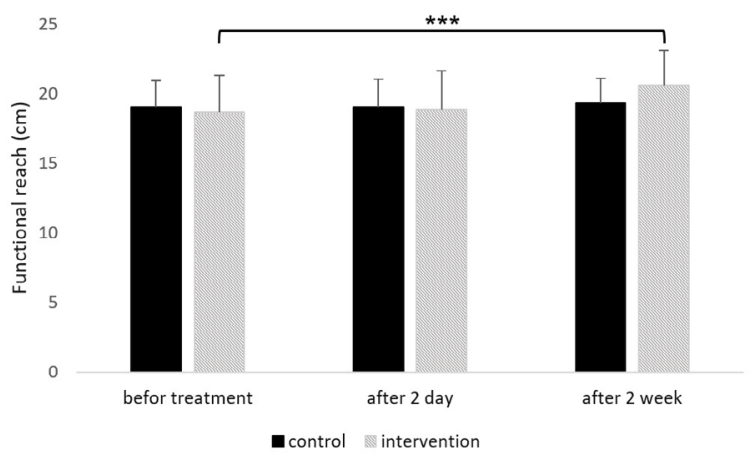

Figure 1. The results of one comparison of means

The results of one comparison of means were significant $(\mathrm{P}<0.01)$ and are shown by stars. ever, the main effects of groups (intervention, control) were not significant for any of the variables. Results of independent t-test showed no significant difference between groups in second $(\mathrm{P}=0.882)$ and third evaluations in FR scores $(\mathrm{P}=0.120)$. Triple evaluations, performed on changes of each group using paired t-test, indicated no significant difference between the first and second evaluations of FR scores in the intervention group $(\mathrm{P}=0.082)$,

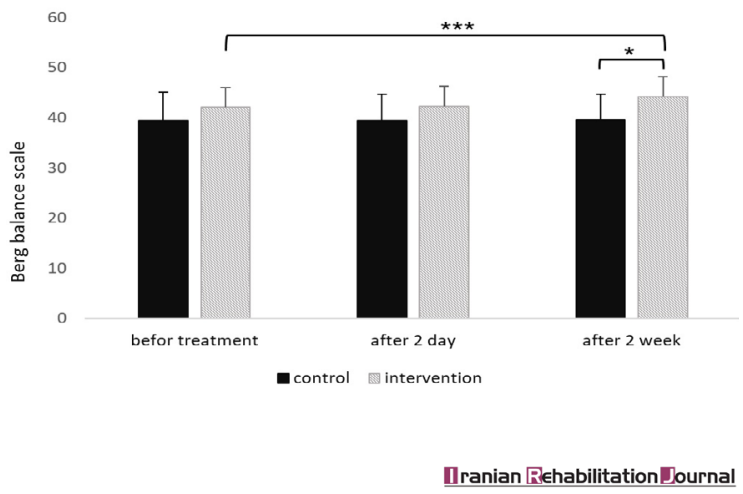

Figure 2. The interaction plot of groups by time of measuring dynamic balance

The results of comparisons of means showed significant difference between significant difference between the groups in the third evaluation $\left({ }^{*} \mathrm{P}<0.05\right)$ and the first and third evaluations in the intervention group $(* * * \mathrm{P}<0.001)$. 
however, a significant difference was observed before and after intervention evaluations $(\mathrm{P}<0.001)$.

In the control group, no significant difference was observed between the first and second evaluations $(\mathrm{P}=0.1)$, and first and third evaluations $(\mathrm{P}=0.1)$ (Figure 1). Results of independent t-test also showed no significant difference between groups in the second $(\mathrm{P}=0.116)$ evaluation but significant difference in the third evaluation in BBS scores between the intervention and control groups $(\mathrm{P}=0.01)$. Paired t-test showed no significant difference between the first and second evaluations of BBS scores in the intervention group $(\mathrm{P}=0.774)$, however, a significant difference was observed between the first and third evaluations of the intervention groups $(\mathrm{P}<0.001)$. In the control group, no significant difference was observed between the first and second evaluations $(\mathrm{P}=0.8)$ and with the first and third evaluations $(\mathrm{P}=0.8)$ (Figure 2$)$.

\section{Discussion}

The current study aimed at analyzing the effects of $\mathrm{Ki}$ nesio tape on static and dynamic balance of children with spastic Cerebral Palsy in three intervals of before, two days after, and two weeks after the start of interventions. After two days, results indicated no significant change in the static and dynamic balance, however, a significant change was observed after two weeks of interventions. It indicated that the variables were improved by Kinesio tape through this time.

Children with spastic Cerebral Palsy have problems in their movements and balance. Weak balance causes some limitations in movement ability and is followed by reduced ability of understanding the surroundings, and communicating with family members and friends $[15,31]$. It can be inferred that Kinesio tape applies a reinforcement tension on ankles and knees in order to improve walking position and the contact of feet with ground $[32,33]$. Therefore, this can lead to improved balance in children with Cerebral Palsy. On the other hand, Kinesio tape induces central nervous sensory system, and in this regard, adjusts muscle functions and spatial-temporal reactions, which consequently leads to reduced postural fluctuations and improved balance [34].

Da Costa et al. conducted a study on instantaneous effects of Kinesio tape for up standing movements, balance, and dynamic control of four children with left hemiplegia. Their results indicated reduced up standing time and timed up and go, which were consistent with the current study results. Comparison before and after intervention balances indicated no significant difference which was not consis- tent with the current study results. The researchers concluded that neuromuscular tape was effective in dynamic activities, but not in static activities [35].

Pour Momeni et al. analyzed the effects of Kinesio tape on balance and spasticity of patients with stroke. During the study, Kinesio tape was used for both groups for one hour. Intergroup results indicated that the Timed Up and Go test (TUG) score and gastrocnemius muscle tone reduced significantly. The intergroup comparison indicated that Kinesio tape was more effective on patients with stroke that had left hemiplegia. These results were consistent with those of the study [36]. Kaya et al. analyzed the effects of Kinesio tape on functions and activities of children with spastic Cerebral Palsy. They concluded that Kinesio taping technique is an alternative approach to improve proprioception, movement functions, and daily activities of children with Cerebral Palsy [37].

Cortesi et al. used Kinesio tape on gastrocnemius muscles of patients with MS. This technique significantly reduced the fluctuations and improved balance in such patients [38]. In another research, consistent with the current study, Losa claimed that Kinesio tape is a positive forward step toward rehabilitation of children with Cerebral Palsy and can improve their static and dynamic balance [26]. Losa et al. proved that using Kinesio tape for ankles and knees of children with Cerebral Palsy can improve their balance and walking. These results were consistent with those of the current study. However, the analysis of spasticity and movement range in the current study indicated that Kinesio tape had no significant effect on them [39].

The small sample size may be considered a potential limitation for the current study findings inference. Moreover, a follow-up is required to evaluate the maintenance effectiveness of Kinesio tape on static and dynamic balance in children with Cerebral Palsy. Lack of evaluating the independence in activities using tests such as functional independence measure to evaluate transfer of learning was another limitation of the current study.

\section{Conclusion}

Based on the results of the current study (long term affectability) and other researches, Kinesio tape is effective on children with spastic diplegia and quadriplegia Cerebral Palsy and can improve their balance. Therefore, Kinesio tape can be used as an alternative method to rehabilitate and treat children with spastic diplegia and quadriplegia Cerebral Palsy. 


\section{Ethical Considerations}

\section{Compliance with ethical guidelines}

The current study was approved by the Ethics Committee of Baqiyatallah University of Medical Sciences (code: IR.BMSU.REC.1396.521). A biographical form was filled by the patient, and an ethical approval form was signed by the patient's companion.

\section{Funding}

The study was conducted with the financial support of Exercise Physiology Research Center, Life Style Institute, Baqiyatallah University of Medical Sciences, Tehran, Iran.

\section{Authors' contributions}

Conceptualization: Mirjavad Tabatabaee, Alireza Shamsoddini; Methodology: Alireza Shamsoddini, Mirjavad Tabatabaee, Moslem Cheraghifard; Investigation: Mirjavad Tabatabaee; Writing original draft: Mirjavad Tabatabaee, Moslem Cheraghifard; Data analysis: Moslem Cheraghifard; Review \& editing: Alireza Shamsoddini, Moslem Cheraghifard; and Supervision: Alireza Shamsoddini.

\section{Conflict of interest}

The authors declare no conflict of interest

\section{References}

[1] A'sgari T, Hadian MR, Nakhostin-Ansari N, Abdolvahhab M, Jalili M, Faghih-Zadeh S. [Berg balance scale reliability for evaluation in children with spastic diplegia (Persian)]. Archives of Rehabilitation. 2007; 8(2):13-6.

[2] Hadian MR, Nakhostin Ansari N, Asgari T, Abdolvahab M, Jalili M. [Inter \& intra rater reliability of Berg Balance scale for evaluation of the balance in children with spastic hemieplegia (Persian)]. Journal of Modern Rehabilitation. 2007; 1(2):31-7.

[3] Graham HK, Selber P. Musculoskeletal aspects of Cerebral Palsy. The Journal of Bone and Joint Surgery British Volume. 2003; 85(2):157-66. [DOI:10.1302/0301-620X.85B2.14066]

[4] Stanley FJ, Blair E, Alberman E. Cerebral palsies: Epidemiology and causal pathways. Cambridge: Cambridge University Press; 2000

[5] Panteliadis CP, Darras BT. Encyclopaedia of paediatric neurology: Theory and practice. Stuttgart: Thieme Medical Publishers; 1999.

[6] Kembhavi G, Darrah J, Magill-Evans J, Loomis J. Using the berg balance scale to distinguish balance abilities in chil- dren with Cerebral Palsy. Pediatric Physical Therapy. 2002 14(2):92-9. [DOI:10.1097/00001577-200214020-00005] [PMID]

[7] Hayes MS, McEwen IR, Lovett D, M'lisa MS, Smith DW. Next step: Survey of pediatric physical therapists' educational needs and perceptions of motor control, motor development and motor learning as they relate to services for children with developmental disabilities. Pediatric Physical Therapy. 1999; 11(4):164-82. [DOI:10.1097/00001577-199901140-00002]

[8] Steultjens EM, Dekker J, Bouter LM, van de Nes JC, Cup $\mathrm{EH}$, van den Ende $\mathrm{CH}$, et al. Occupational therapy for stroke patients: When, where, and how? Stroke. 2003; 34(3):676-87. [DOI:10.1161/01.STR.0000057576.77308.30] [PMID]

[9] Mazzone S, Serafini A, Iosa M, Aliberti M, Gobbetti T, Paolucci $\mathrm{S}$, et al. Functional taping applied to upper limb of children with hemiplegic Cerebral Palsy: A pilot study. Neuropediatrics. 2011; 42(06):249-53. [DOI:10.1055/s-0031-1295478] [PMID]

[10] Franjoine MR, Darr N, Held SL, Kott K, Young BL. The performance of children developing typically on the pediatric balance scale. Pediatric Physical Therapy. 2010; 22(4):350-9. [DOI:10.1097/PEP.0b013e3181f9d5eb] [PMID]

[11] Amirsalari S, Dalvand H, Dehghan L, Feizy A, Hosseini SA, Shamsodini A. [The efficacy of botulinum toxin type A injection in the hamstring and calf muscles with and without serial foot casting in gait improvement in children with Cerebral Palsy (Persian)]. Tehran University Medical Journal. 2011; 69(8):509-17.

[12] Yasukawa A, Patel P, Sisung C. Pilot study: Investigating the effects of Kinesio Taping ${ }^{\circledR}$ in an acute pediatric rehabilitation setting. American Journal of Occupational Therapy. 2006; 60(1):104-10. [DOI:10.5014/ajot.60.1.104] [PMID]

[13] Taylor RL, O'brien L, Brown T. A scoping review of the use of elastic therapeutic tape for neck or upper extremity conditions. Journal of Hand Therapy. 2014; 27(3):235-46. [DOI:10.1016/j.jht.2014.03.004] [PMID]

[14] Skirven TM, Osterman AL, Fedorczyk J, Amadio PC. Rehabilitation of the hand and upper extremity, 2-volume set e-book Expert consult. Amsterdam: Elsevier Health Sciences; 2011.

[15] Harada N, Chiu V, Damron-Rodriguez J, Fowler E, Siu A, Reuben DB. Screening for balance and mobility impairment in elderly individuals living in residential care facilities. Physical Therapy. 1995; 75(6):462-9. [DOI:10.1093/ptj/75.6.462] [PMID]

[16] Bell KJ, Ounpuu S, DeLuca PA, Romness MJ. Natural progression of gait in children with Cerebral Palsy. Journal of Pediatric Orthopaedics. 2002; 22(5):677-82. [DOI:10.1097/00004694-200209000-00020] [PMID]

[17] Charles J, Gordon AM. A critical review of constraintinduced movement therapy and forced use in children with hemiplegia. Neural Plasticity. 2005; 12(2-3):245-61 [DOI:10.1155/NP.2005.245] [PMID] [PMCID]

[18] Cherng RJ, Su FC, Chen JJ, Kuan TS. Performance of static standing balance in children with spastic diplegic Cerebral Palsy under altered sensory environments. American Journal of Physical Medicine \& Rehabilitation. 1999; 78(4):336-43 [DOI:10.1097/00002060-199907000-00008]

[19] Yi SH, Hwang JH, Kim SJ, Kwon JY. Validity of pediatric balance scales in children with spastic Cerebral Palsy. Neuropediatrics. 2012; 43(6):307-13. [DOI:10.1055/s-0032-1327774] [PMID] 
[20] Pountney TE, Mulcahy C, Green E. Early development of postural control. Physiotherapy. 1990; 76(12):799-802. [DOI:10.1016/S0031-9406(10)63184-7]

[21] Chen CL, Shen IH, Chen CY, Wu CY, Liu WY, Chung CY. Validity, responsiveness, minimal detectable change, and minimal clinically important change of Pediatric Balance Scale in children with Cerebral Palsy. Research in Developmental Disabilities. 2013; 34(3):916-22. [DOI:10.1016/j. ridd.2012.11.006] [PMID]

[22] Iosa M, Fusco A, Morone G, Pratesi L, Coiro P, Venturiero $\mathrm{V}$, et al. Assessment of upper-body dynamic stability during walking in patients with subacute stroke. Journal of Rehabilitation Research \& Development. 2012; 49(3):439-50. [DOI:10.1682/JRRD.2011.03.0057] [PMID]

[23] Allum J, Bloem B, Carpenter M, Hulliger M, HaddersAlgra M. Proprioceptive control of posture: A review of new concepts. Gait \& Posture. 1998; 8(3):214-42. [DOI:10.1016/ S0966-6362(98)00027-7]

[24] Mackin EJ, Callahan AD, Osterman AL, Schneider LH, Skirven TM. Hunter Mackin callahan-rehabilitation of the hand and upper extremity. Maryland Heights: Mosby; 2002.

[25] Kuo YL, Huang YC. Effects of the application direction of Kinesio taping on isometric muscle strength of the wrist and fingers of healthy adults-a pilot study. Journal of Physical Therapy Science. 2013; 25(3):287-91. [DOI:10.1589/jpts.25.287]

[26] Iosa M. The application of Kinesio Taping in children with Cerebral Palsy. Developmental Medicine \& Child Neurology. 2015; 57(1):11-2. [DOI:10.1111/dmcn.12601] [PMID]

[27] Śliwiński Z, Halat B, Kufel W, Michalak B, Kiljański M. The effect of Kinesio Taping applications on motor activity in children with developmental defects. Fizjoterapia Polska. 2007; 7(1):52-62.

[28] Shamsoddini A, Rasti Z, Kalantari M, Hollisaz MT, Sobhani $V$, Dalvand $H$, et al. The impact of Kinesio taping technique on children with Cerebral Palsy. Iranian Journal of Neurology. 2016; 15(4):219-27. [PMID] [PMCID]

[29] Azad A, Taghizadeh G, Khaneghini A. Assessments of the reliability of the Iranian version of the Berg Balance scale in patients with multiple sclerosis. Acta Neurologica Taiwanica. 2011; 20(1):22-8

[30] Duncan PW, Weiner DK, Chandler J, Studenski S. Functional reach: A new clinical measure of balance. Journal of Gerontology. 1990; 45(6):M192-M7. [DOI:10.1093/ geronj/45.6.M192]

[31] Labaf S, Shamsoddini A, Hollisaz MT, Sobhani V, Shakibaee A. Effects of neurodevelopmental therapy on gross motor function in children with Cerebral Palsy. Iranian Journal of Child Neurology. 2015; 9(2):36-41. [PMID] [PMCID]

[32] Booth B, Doyle M, Montgomery J. Serial casting for the management of spasticity in the head-injured adult. Physical Therapy. 1983; 63(12):1960-6. [DOI:10.1093/ptj/63.12.1960] [PMID]

[33] Griffin JW. Use of proprioceptive stimuli in therapeutic exercise. Physical Therapy. 1974; 54(10):1072-9. [DOI:10.1093/ $\mathrm{ptj} / 54.10 .1072]$ [PMID]

[34] Shumway-Cook A. Normal postural control. Motor control: Theory and practical applications. Philadelphia: Lippincott Williams \& Wilkins; 2001.
[35] Added MAN, Costa LOP, Fukuda TY, De Freitas DG, Salomão EC, Monteiro RL, et al. Efficacy of adding the kinesio taping method to guideline-endorsed conventional physiotherapy in patients with chronic nonspecific low back pain: A randomised controlled trial. BMC Musculoskeletal Disorders. 2013; 14(1):301. [DOI:10.1186/1471-2474-14-301] [PMID] [PMCID]

[36] Jalaee F, Pourmomeni A, Karimi M, Baharlouei H. [Effects of gastronomies kinesio taping on postural control and spasticity in patients with chronic stroke (Persian)]. Journal of Isfahan Medical School. 2015; 33(329):467-78.

[37] Kaya Kara O, Atasavun Uysal S, Turker D, Karayazgan S, Gunel MK, Baltaci G. The effects of Kinesio taping on body functions and activity in unilateral spastic Cerebral Palsy: A single-blind randomized controlled trial. Developmental Medicine \& Child Neurology. 2015; 57(1):81-8. [DOI:10.1111/ dmcn.12583] [PMID]

[38] Cortesi M, Cattaneo D, Jonsdottir J. Effect of Kinesio taping on standing balance in subjects with multiple sclerosis: A pilot studym. NeuroRehabilitation. 2011; 28(4):365-72 [DOI:10.3233/NRE-2011-0665] [PMID]

[39] Iosa M, Morelli D, Nanni MV, Veredice C, Marro T, Medici A, et al. Functional taping: A promising technique for children with Cerebral Palsy. Developmental Medicine \& Child Neurology. 2010; 52(6):587-9. [DOI:10.1111/j.14698749.2009.03539.x] [PMID] 
This Page Intentionally Left Blank 